\title{
Efficient Simulation using Shadowing Fields of Many Wireless Interferers with Correlated Shadowing
}

\author{
Sebastian S. Szyszkowicz, Furkan Alaca, and Halim Yanikomeroglu \\ Department of Systems and Computer Engineering \\ Carleton University \\ Ottawa, Ontario, K1S 5B6, Canada \\ Email: \{sz, falaca, halim\}@sce.carleton.ca
}

\author{
John S. Thompson \\ Institute for Digital Communications \\ University of Edinburgh \\ Edinburgh, EH9 3JL, United Kingdom \\ Email: john.thompson@ed.ac.uk
}

\begin{abstract}
As the number of wireless devices sharing a radio band increases, so does the number $N$ of potential co-channel interferers. The receiver performance is then strongly dependent on the total received interference power. While the statistics of this power have previously been studied under the channel assumption of independent shadowing, it is easy to show that for large $N$ the correlation among the shadowing paths cannot be neglected. While this correlation may be simulated by matrix decomposition, we show that an alternative approximately equivalent algorithm using shadowing fields can achieve simulation performance that scales much better with $N$ and has additional advantages.
\end{abstract}

Index Terms-co-channel interference, correlated shadowing, algorithm optimisation

\section{INTRODUCTION}

In the last few years, the simulation and analysis of interference caused by $N$ distinct co-channel interferers, with $N$ even in the hundreds [1], [2], has received significant interest. The interference from a Poisson field of interferers, a very similar problem, has been studied at least since [3], and still receives attention today [4]. The nature of the interfering nodes may be femtocells [1], sensor nodes, or any other devices that aggressively share spectrum, often in a noncoordinated and opportunistic manner. This type of scenario is becoming increasingly relevant as wireless communications move away from the traditional coordinated cellular model to more heterogeneous and distributed paradigms, such as adhoc networking and cognitive radio [4]. Thus the study of interference from many co-channel interferers is essential for the design of future wireless systems.

We notice however that the modeling in these types of work, while incorporating wireless shadowing, usually does not consider correlation between the shadowing paths [4]. We will demonstrate that the independence assumption, while already known to give different results for small $N$ [5], gives very inaccurate results for large $N$, indicating that shadowing correlation should be incorporated.

A significant problem in the simulation of many paths with correlated shadowing is the computation time: specifically, the most conventional approach [6], [7], Cholesky factorisation, will require a computational time that scales as $N^{3}$ [8], and a memory usage of the order of $N^{2}$ for storing the correlation matrices. This paper demonstrates a Monte Carlo simulation algorithm with fast execution time and reasonable memory usage, both of which scale linearly with increasing $N$; thus the algorithm is useful for simulating future wireless systems with always increasing spectrum reuse, which usually implies an increase in the number of significant interferers.

In Section II, we describe the physical model to simulate. In Section III, we argue why it is unrealistic to neglect shadowing correlation when $N$ is large. In Section IV, we describe both the conventional and the proposed algorithm for simulating interference with correlated shadowing. In Section V, we run simulations using both algorithms and examine their time performance and accuracy. We conclude in Section VI.

\section{Physical Model}

\section{A. Physical Assumptions}

The following are often assumed in interference analysis:

1) Given fixed propagation paths, the logarithm of the shadowing on each path is well-modeled by jointly Gaussian random variables. [5]-[7], [9], [10]

2) The total interference power is the sum of the individual interference powers, as explained by incoherent signal addition. [5], [7], [9], [11]

\section{B. Interference Modeling}

Consider $N$ interferers located at $\vec{r}_{i}\left(r_{i}=\left\|\vec{r}_{i}\right\|\right)$, distributed randomly, independently and identically, according to a valid density function $g(\vec{r})$, such that, without loss of generality, the receiver is located at the origin. Consider power law average pathloss with exponent $\beta$. To simplify the problem, we assume each interferer transmits with equal unit power. Finally, consider $S_{i}$ the shadowing experienced on path $i$. Assume that the vector $\vec{S}=\left[S_{i}\right]_{i=1}^{N}$ is Gaussian when conditioned on $\vec{r}_{1}, \ldots, \vec{r}_{N}$, with 


$$
\begin{aligned}
\mathbb{E}\left\{S_{i}\right\} & =0, \\
\mathbb{E}\left\{S_{i}^{2}\right\} & =\sigma_{\mathrm{s}}^{2}, \\
\mathbb{E}\left\{S_{i} S_{j} \mid \vec{r}_{i}, \vec{r}_{j}\right\} & =\sigma_{\mathrm{s}}^{2} h\left(\vec{r}_{i}, \vec{r}_{j}\right), i \neq j,
\end{aligned}
$$

where $h$ is the shadowing correlation model. Then, the correlation matrix of $\vec{S}$ conditioned on $\vec{r}_{1}, \ldots, \vec{r}_{N}$ is given by

$$
\mathbf{K}_{N \times N}=\sigma_{\mathrm{s}}^{2}\left[\rho_{i, j}\right], \quad \rho_{i, j}= \begin{cases}h\left(\vec{r}_{i}, \vec{r}_{j}\right) & i \neq j, \\ 1 & i=j .\end{cases}
$$

Assuming that $h$ is such that $\mathbf{K}$ is always a positive semidefinite matrix, it follows that the Gaussian vector $\vec{S}$ is always feasible [10], i.e., it can always be constructed, and is fully determined by (1). We are then interested in finding the statistics of the total interference power given by

$$
I=\sum_{i=1}^{N} I_{i}, \quad I_{i}=c r_{i}^{-\beta} e^{\lambda S_{i}},
$$

where $\lambda=0.1 \ln 10$ and $c$ is the common constant gain accounting for multiplicative constants such as antenna gains, reference distance, and transmit power. Without loss of generality, we set $c=1$.

\section{Choice of Correlation Model}

Consider the minimal angle of arrival separation

$$
\theta=\left|\angle \vec{r}_{i}-\angle \vec{r}_{j}\right| \in\left[0^{\circ}, 180^{\circ}\right]
$$

and the arrival distance ratio in $\mathrm{dB}$

$$
R=\left|10 \log _{10} r_{i} / r_{j}\right|=\frac{10}{\ln 10}\left|\ln r_{i}-\ln r_{j}\right| .
$$

We choose a correlation model $h$ that may be expressed in terms of $\theta$ and $R$ only, and that is separable with respect to these dimensions. From the more general class of shadowing correlation models given in [6], we propose using the following subset:

$$
\begin{aligned}
h_{\Theta}(\theta) & = \begin{cases}1-\theta / \theta_{0} & \theta \leq \theta_{0}, \\
0 & \theta>\theta_{0} .\end{cases} \\
h_{\mathrm{R}}(R) & = \begin{cases}1-R / R_{0} & R \leq R_{0}, \\
0 & R>R_{0},\end{cases} \\
h\left(r_{i}, r_{j}\right) & =h_{\Theta}(\theta) h_{\mathrm{R}}(R),
\end{aligned}
$$

with tunable parameters $0^{\circ}<\theta_{0} \leq 180^{\circ}$ and $R_{0}>0$.

We chose this model among many others for the following reasons:

1) In [10], we have shown that this model always yields positive semidefinite correlation matrices $\mathbf{K}$. This is not the case for several of the existing models.

2) Furthermore in [10], we have argued that from among all models that always give positive semidefinite correlation matrices $\mathbf{K}$, this was the model that seemed most physically realistic.

3) The selected model has two tunable parameters, and can therefore approximate a wide range of correlation models with reasonable accuracy, as done in [6].

4) The mathematical form of this model lends itself particularly well to fast simulation using shadowing fields, as we will demonstrate in Section IV.

\section{Statistical BehaViour For MANy InTERFERERS}

In interference analysis, we are interested is studying the statistical behaviour of the total interference power $I$. The natural approach is first to establish its mean and variance:

$$
\begin{aligned}
A & =e^{\frac{1}{2} \lambda^{2} \sigma_{\mathrm{s}}^{2}} \mathbb{E}\left\{r_{1}^{-\beta}\right\}, \\
B & =e^{2 \lambda^{2} \sigma_{\mathrm{s}}^{2}} \mathbb{E}\left\{r_{1}^{-2 \beta}\right\}, \\
C & =\mathbb{E}\left\{e^{\lambda\left(S_{1}+S_{2}\right)} r_{1}^{-\beta} r_{2}^{-\beta}\right\}, \\
\mathbb{E}\{I\} & =N A, \\
\mathbb{E}\left\{I^{2}\right\} & =N B+\left(N^{2}-N\right) C, \\
\mathbb{V A R}\{I\} & =N(B-C)+N^{2}\left(C-A^{2}\right) .
\end{aligned}
$$

We observe that, while for independent shadowing we have $C=A^{2} \Rightarrow \operatorname{VAR}\{I\}=\mathcal{O}(N)$, in general for correlated shadowing $\operatorname{VAR}\{I\}=\mathcal{O}\left(N^{2}\right)$. The mean power of $I$ remains the same regardless of correlation. Therefore what was already observed for small $N$ [5] will be even more significant for large $N$ : adding correlation changes (specifically, broadens) the distribution of $I$ significantly. It follows that, given a sufficiently realistic shadowing correlation model, the distribution of $I$ obtained using correlated shadowing will be much more realistic than that obtained using independent shadowing.

Also, because of the asymptotic behaviour of the mean and variance, analysing $I$ as $N \rightarrow \infty$ requires the study of the convergence of $I / N$ (rather than $(I-\mathbb{E}\{I\}) / \sqrt{N}$ in the independent case). Because of the existence of correlation, the classical Central Limit Theorem cannot be applied for large $N$. Indeed $I / N$ does not necessarily converge to a Gaussian distribution, and may in fact converge to a distribution close to a lognormal with large spread as we have observed in [7].

We can therefore conclude that correlation in shadowing becomes a dominating factor in the distribution of $I$ as $N$ becomes large.

\section{Simulation of Correlated Shadowing}

In order to simulate $I$ (and, in fact, $I_{i}$ ), there are two very different approaches that nevertheless can give very close results, namely matrix factorisation and shadowing fields. A simulation can be fully specified by the parameters listed in Table I.

\section{A. Matrix Factorisation}

Generating $\vec{S}$ in Monte Carlo simulations is traditionally [6] done by solving for $\mathbf{C}_{N \times N}$ in the equation

$$
\mathbf{K}=\mathbf{C}^{T} \mathbf{C} \text {, }
$$

for each particular realisation of $\mathbf{K}$. We will write $\mathbf{C}=\sqrt[*]{\mathbf{K}}$. The next step is to generate a vector $\vec{Z}=\left[Z_{i}\right]_{i=1}^{N}$ of independent standard Gaussian $\mathcal{N}(0,1)$ random variables. $\vec{S}$ is then obtained from

$$
\vec{S}=\vec{Z} \mathbf{C} .
$$


TABLE I

SiMUlation PARAMETERS

\begin{tabular}{|c|c|}
\hline Parameter & Description \\
\hline 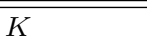 & Total number of Monte Carlo trials of $I$ \\
\hline$N$ & Number of interferers \\
\hline \multicolumn{2}{|l|}{ Physical } \\
\hline$g(\vec{r})$ & Statistical density of interferers' positions \\
\hline$r_{\min }, r_{\max }$ & $\begin{array}{l}\text { Limits on interferers' distances from receiver: } \\
g(\vec{r})=0 \forall \vec{r}:\|\vec{r}\| \notin\left[r_{\min }, r_{\text {max }}\right]\end{array}$ \\
\hline$\beta$ & Pathloss exponent \\
\hline$\sigma_{\mathrm{s}}$ & Shadowing standard deviation in $\mathrm{dB}$ \\
\hline$\theta_{0}, R_{0}$ & Parameters of shadowing correlation model (6) \\
\hline \multicolumn{2}{|c|}{$\underline{\text { Shadow Fields }}$} \\
\hline$D_{\Theta}$ & Number of discrete field points in $\theta$ dimension \\
\hline$D_{\mathrm{R}}$ & Number of discrete field points in $R$ dimension \\
\hline$F_{\Theta}$ & $\begin{array}{l}\text { Length of digital FIR filter in } \theta \text { dimension } \\
=\text { nearest integer to } D_{\Theta} \theta_{0} / 2 \pi\end{array}$ \\
\hline$F_{\mathrm{R}}$ & $\begin{array}{l}\text { Length of digital FIR filter in } R \text { dimension } \\
=\text { nearest integer to } D_{\mathrm{R}} R_{0} / 10 \log _{10}\left(r_{\max } / r_{\min }\right)\end{array}$ \\
\hline $\mathbf{F}$ & $\begin{array}{l}F_{\Theta} \times F_{\mathrm{R}} \text { filtering kernel } \\
=\text { constant } 1 / \sqrt{F_{\Theta} F_{\mathrm{R}}} \text { for model }(6)\end{array}$ \\
\hline
\end{tabular}

This is implemented as follows:

\section{MATRIX FACTORISATION ALGORITHM}

Ensure: The histogram of $I[k]$ approximates the $p d f$ of $I$.

$$
\begin{aligned}
& \text { for } k=1 \text { to } K \text { do } \\
& \text { for } i=1 \text { to } N \text { do } \\
& \vec{r}_{i} \Leftarrow i . i . d \text {. random from } g(\vec{r}) \\
& Z_{i} \Leftarrow i . i . d \text {. random } \mathcal{N}(0,1) \\
& K[i, i] \Leftarrow \sigma_{\mathrm{s}}^{2} \\
& \text { for } j=1 \text { to } i-1 \text { do } \\
& K[i, j]=K[j, i] \Leftarrow \sigma_{\mathrm{s}}^{2} h\left(\vec{r}_{i}, \vec{r}_{j}\right) \\
& \text { end for } \\
& \text { end for } \\
& \mathbf{C} \leftarrow \sqrt[*]{\mathbf{K}} \\
& \vec{S} \leftarrow \vec{Z} \mathbf{C} \\
& I[k] \Leftarrow \sum_{i=1}^{N} e^{\lambda S_{i}} r_{i}^{-\beta} \\
& \text { end for }
\end{aligned}
$$

Solving $\sqrt[*]{\mathbf{K}}$ can be performed efficiently by Cholesky factorisation ${ }^{1}$ with complexity $\mathcal{O}\left(N^{3}\right)$ [7], [8].

\section{B. Shadowing Fields}

A shadowing field is a random process in two dimensions (properly, a random field), such that it is a Gaussian process with a specific autocorrelation function. This autocorrelation is such that, when interferers with positions $\vec{r}_{i}$ are placed on the field, and the value of the field at the point $\vec{r}_{i}$ is taken as the value of $S_{i}$, then $\vec{S} \mid \vec{r}_{1}, \ldots, \vec{r}_{N}$ has the desired correlation matrix $\mathbf{K}$. This can be compared by analogy to gravitational

\footnotetext{
${ }^{1}$ While Cholesky factorisation fails for singular matrices $\mathbf{K}$ [8], [10], we have observed in simulations here and in [7] that this event is extremely rare using model (6) with double-precision arithmetic.
}

(electric, etc.) fields, where the field gives the acceleration of a mass placed at any point, whether there actually is a mass at that point or not.

The idea of generating shadowing fields has already been explored [9], [12]-[14] with correlation functions of the form $h\left(\vec{r}_{i}, \vec{r}_{j}\right)=f\left(\left\|\vec{r}_{i}-\vec{r}_{j}\right\|\right)$. We have argued in [10] that such models may not reflect true shadowing spatial correlation characteristics. Furthermore, correlation as a separable function of $\theta$ and $R$ can be easily simulated by using a geometric transformation. The accuracy of our method is limited only by the quantisation level.

Consider a random field (a two-dimensional random process) $\overline{\mathbf{M}}$ of continuous parameters $(x, y)$. Let the random field be stationary [15], with an auto-correlation function such that the correlation between the field at two points $\overline{\mathbf{M}}\left(x_{i}, y_{i}\right)$ and $\overline{\mathbf{M}}\left(x_{j}, y_{j}\right)$ correspond to the desired shadowing correlation under some transformation.

Consider what we will call the log-polar ${ }^{2}$ transformation:

$$
\begin{aligned}
\mathcal{T}_{\mathrm{LP}}:(\theta, R) & \longmapsto 10^{0.1 R}(\cos \theta, \sin \theta), \\
\mathcal{T}_{\mathrm{LP}}:[0,2 \pi] & \times\left[10 \log _{10} r_{\min }, 10 \log _{10} r_{\max }\right] \\
& \longmapsto\left\{\vec{r}: r_{\min } \leq r \leq r_{\max }\right\} .
\end{aligned}
$$

Let us choose the autocorrelation of $\overline{\mathbf{M}}$ as

$$
\begin{aligned}
& \eta_{x}(\xi)= \begin{cases}1-|\xi| / \theta_{0} & |\xi| \leq \theta_{0}, \\
0 & \theta_{0} \leq|\xi| \leq 2 \pi-\theta_{0}, \\
1+(|\xi|-2 \pi) / \theta_{0} & 2 \pi-\theta_{0} \leq|\xi| \leq 2 \pi .\end{cases} \\
& \eta_{y}(v)= \begin{cases}1-|v| / R_{0} & |v| \leq R_{0}, \\
0 & |v|>R_{0},\end{cases} \\
& \mathbb{E}\{\overline{\mathbf{M}}(x+\xi, y+v) \overline{\mathbf{M}}(x, y)\}=\eta_{x}(\xi) \eta_{y}(v),
\end{aligned}
$$

for $\overline{\mathbf{M}}$ defined on $[0,2 \pi] \times\left[10 \log _{10} r_{\min }, 10 \log _{10} r_{\max }\right]$. We find that the field $\overline{\mathbf{M}}$, under transformation $\mathcal{T}_{\mathrm{LP}}$, has the correlation properties of (6), i.e.,

$$
\begin{aligned}
& \mathcal{T}_{\mathrm{LP}}:\left(x_{i}, y_{i}\right) \longmapsto \vec{r}_{i}, i=1,2, \ldots \\
& \mathbb{E}\left\{\overline{\mathbf{M}}\left(x_{1}, y_{1}\right) \overline{\mathbf{M}}\left(x_{2}, y_{2}\right)\right\}=h\left(\vec{r}_{1}, \vec{r}_{2}\right) .
\end{aligned}
$$

Therefore, we may write

$$
S_{i}=\sigma_{\mathrm{s}} \overline{\mathbf{M}}\left(\mathcal{T}_{\mathrm{LP}}^{-1}\left(\vec{r}_{i}\right)\right),
$$

and $S_{i}$ 's will have the same correlation matrix as in (9).

For numerical purposes, $\overline{\mathbf{M}}$ can be approximated by a discrete-parameter matrix $\mathbf{M}_{D_{\Theta} \times D_{\mathrm{R}}}$, with a regularly-spaced quantisation grid along $\theta$ and $R$. Correlation of the form (6), triangular in both dimensions, can be obtained by using a uniform square filter $\mathbf{F}_{F_{\Theta} \times F_{\mathrm{R}}}$, ideally choosing $F_{\Theta}$ and $F_{\mathrm{R}}$ so that we have exactly

$$
\begin{aligned}
& F_{\Theta} / D_{\Theta}=\theta_{0} / 2 \pi, \\
& F_{\Theta} / D_{\Theta}=R_{0} / 10 \log _{10}\left(r_{\max } / r_{\min }\right),
\end{aligned}
$$

with $\mathbf{F}$ equal everywhere to $1 / \sqrt{F_{\Theta} F_{\mathrm{R}}}$.

\footnotetext{
${ }^{2} \mathrm{~A}$ polar representation of shadowing fields is suggested in [12].
} 
To obtain the value of the discretised field $\mathbf{M}$ at some point, we must round the coordinates $\mathcal{T}_{\mathrm{LP}}^{-1}\left(\vec{r}_{i}\right)$ to the nearest quantisation point. Therefore the algorithm is limited in precision by the finite spatial quantisation. On the other hand, the computational cost of generating one field grows $\mathcal{O}\left(F_{\Theta} F_{\mathrm{R}} D_{\Theta} D_{\mathrm{R}}\right)=\mathcal{O}\left(D_{\Theta}{ }^{2} D_{\mathrm{R}}{ }^{2}\right)$, and so it is critical to choose the number of quantisation points $D_{\Theta} D_{\mathrm{R}}$ properly to balance precision and computational time.

\section{Efficient Filtering for Triangular Correlation Functions}

1) Separability: The nature of the correlation model (6) is such that it can be expressed as the product of a function of $\theta$ and a function of $R$. It follows [15] that the resulting two-dimensional process in the $\theta-R$ plane is also separable. It can therefore be simulated by filtering over each dimension separately, which reduces the general filtering cost from $\mathcal{O}\left(F_{\Theta} F_{\mathrm{R}} D_{\Theta} D_{\mathrm{R}}\right)$ (as in, e. g., [13]) to $\mathcal{O}\left(\left(F_{\Theta}+F_{\mathrm{R}}\right) D_{\Theta} D_{\mathrm{R}}\right)$.

2) Optimised Box Filters: The triangular form in $\theta$ and $R$ of the correlation expression in (6) requires the use of rectangular (box) filters applied to a white Gaussian process. Computationally this is very efficient, as the filtering requires no multiplications. Additionally, it can be implemented even more efficiently [16], with the number of additions now approximately $2 D_{\Theta}$ and $2 D_{\mathrm{R}}$ in each respective dimension, rather than $\left(F_{\Theta}-1\right) D_{\Theta}$ and $\left(F_{\mathrm{R}}-1\right) D_{\mathrm{R}}$ respectively. This is due to the fact that adjacent outputs of a box filter differ only by two input values. This makes the total computation cost for one field realisation $\mathcal{O}\left(D_{\Theta} D_{\mathrm{R}}\right)$, which is independent of the filter size, and hence of the correlation distances $\theta_{0}, R_{0}$.

3) Optimised Shadow Fields Algorithm: Applying separability and optimised box filters gives the following algorithm:

\section{Basic Shadowing Field Algorithm}

Ensure: The histogram of $I[k]$ approximates the $p d f$ of $I$.

$$
\begin{gathered}
\text { for } k=1 \text { to } K \text { do } \\
\text { for } i=1 \text { to } N \text { do } \\
\vec{r}_{i} \Leftarrow i . i . d \text {. random from } \\
\text { end for } \\
\mathbf{M} \Leftarrow \text { shadowing field real } \\
\text { for } i=1 \text { to } N \text { do } \\
S_{i} \leftarrow \sigma_{\mathrm{s}} \mathbf{M}\left[\mathcal{T}_{\mathrm{LP}}^{-1}\left(\vec{r}_{i}\right)\right] \\
\text { end for } \\
I[k] \Leftarrow \sum_{i=1}^{N} e^{\lambda S_{i}} r_{i}^{-\beta} \\
\text { end for }
\end{gathered}
$$$$
\vec{r}_{i} \Leftarrow i . i . d \text {. random from } g(\vec{r})
$$$$
\mathbf{M} \Leftarrow \text { shadowing field realisation }^{\dagger} \text {. }
$$

The Shadowing field for the correlation model (6) can be obtained efficiently [16] in the following manner:

\section{${ }^{\dagger}$ Fast Shadowing Field Generation}

Ensure: $\mathbf{M}$ is Gaussian, correlated approximately as (6).

$$
\mathbf{Z}_{D_{\Theta} \times\left(D_{\mathrm{R}}+F_{\mathrm{R}}-1\right)} \Leftarrow \text { i.i.d. random } \mathcal{N}(0,1)
$$

Initialise a temporary matrix $\mathbf{W}_{D_{\Theta} \times\left(D_{\mathrm{R}}+F_{\mathrm{R}}-1\right)}$

for $m=1$ to $D_{\mathrm{R}}+F_{\mathrm{R}}-1$ do

$\mathbf{W}[1, m] \Leftarrow \sum_{n=1}^{F_{\Theta}} \mathbf{Z}[n, m]$

end for

for $n=1$ to $D_{\Theta}-1$ do

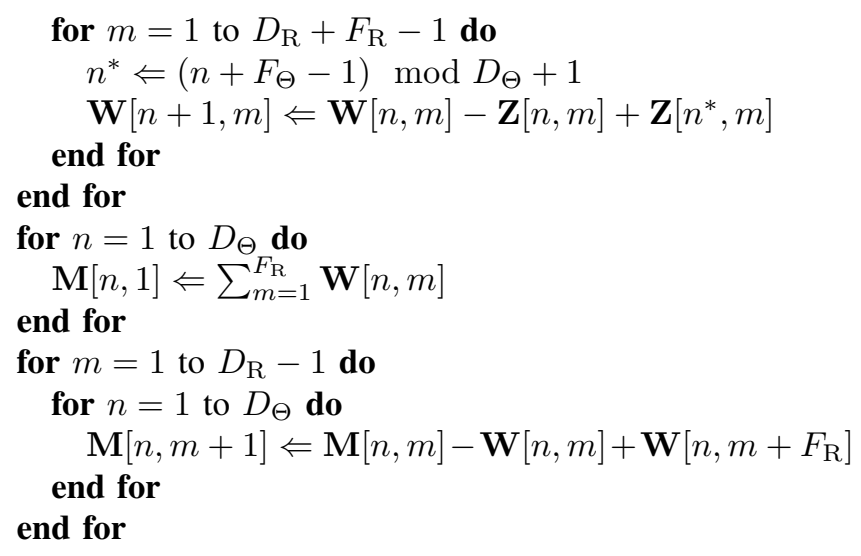

\section{Algorithm COMPARISON THROUGH Simulation}

We now evaluate the performance of the two algorithms in obtaining an estimate of the distribution of $I$. Matrix factorisation is considered to be the benchmark algorithm, because it is an exact realisation of the model described in Section II as $K \rightarrow \infty$. We perform Monte Carlo Simulations using the parameters listed in Table II. In Figure 1, cumulative distribution functions obtained from both algorithms are plotted on lognormal paper [7], [11], which illustrates well all parts of the distribution. We observe that the two algorithms give estimates of the distribution that are well within $2 \mathrm{~dB}$ of each other (horizontally). Additionally, we plot what the distribution would be if $S_{i}$ 's were independent, and we see the widening gap as $N$ increases, as predicted by (7).

We then measure the running time of these simulations for varying $N$. We consider that the time required to perform the matrix factorisation algorithm for $N=1$ is the baseline for simulation time (in our case, $\approx 70$ seconds), which depends strongly on implementation, and we thus consider only the excess execution time above the baseline. We plot these execution times in Figure 2, where we observe that generating shadowing fields outperforms matrix factorisation for approximately $N \geq 30$. An initial investment of time (here $\approx 56+70$ seconds) is required to generate the fields, after which the required time grows slowly with $N$. On the other hand, matrix factorisation becomes prohibitive for large $N$.

Execution time will of course vary with hardware and implementation, but this comparison indicates that the execution times for both algorithms grow at a different rate, and therefore shadowing fields will always outperform matrix factorisation for large enough $N$. These simulations were performed in The MathWorks MATLAB version 7.6.0.324 on Microsoft Windows XP (2002), on a $3.16 \mathrm{GHz}$ Intel Core2 Duo CPU with $3.25 \mathrm{~GB}$ of RAM.

\section{CONCLUSION}

We have shown that shadowing fields can accurately approximate the distribution of the total interference power, and do so in reasonable execution time when the number of interferers becomes large. We have shown that, as this number increases, shadowing correlation becomes a dominating factor 
TABLE II

SiMULATION SETTINGS

\begin{tabular}{ll}
\hline Parameter & Value \\
\hline \hline$K$ & 1000000 \\
$g(\vec{r})$ & uniform over an annular region with radii: \\
$r_{\min }$ & 50 \\
$r_{\max }$ & 500 \\
$\beta$ & 4 \\
$\sigma_{\mathrm{s}}$ & $10 \mathrm{~dB}$ \\
$\theta_{0}$ & $60^{\circ}$ \\
$R_{0}$ & $6 \mathrm{~dB}$ \\
$D_{\Theta}$ & 30 \\
$D_{\mathrm{R}}$ & 25 \\
$F_{\Theta}$ & 5 \\
$F_{\mathrm{R}}$ & 15 \\
\hline
\end{tabular}

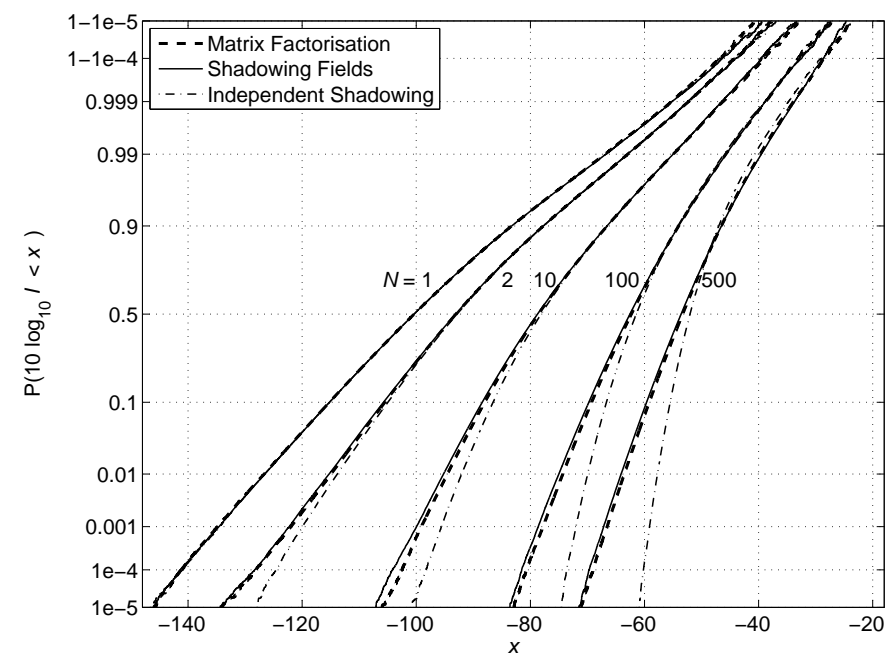

Fig. 1. Monte-Carlo approximations of the distribution of $I$ using both algorithms.

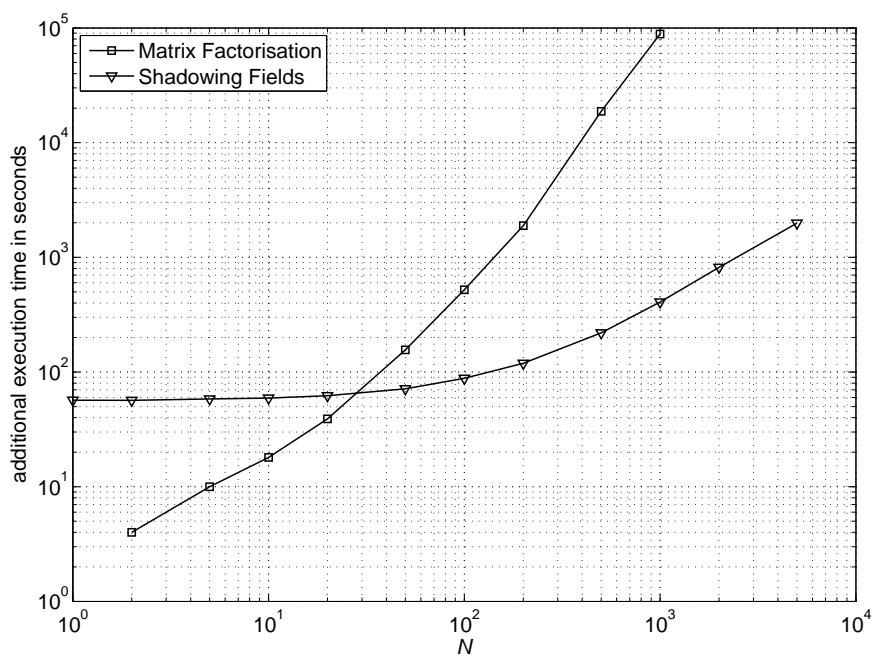

Fig. 2. Execution time for both algorithms, minus base time of 70 s for Matrix Factorisation with $N=1$. and needs to be included in the simulation and analysis of future interference-intensive scenarios.

The correlation model used has good mathematical and physical properties, is flexible, and proves to be easy to implement using shadowing fields. However, other models, particularly if separable, can be used along the same lines.

Also, the simulations can easily be extended to include other factors such as variable transmit power and small-scale fading.

Shadowing fields have the additional advantages of requiring only $\mathcal{O}(N)$ memory (rather than $\mathcal{O}\left(N^{2}\right)$ for matrix factorisation), and being able to accommodate interferer mobility easily: indeed, while matrix factorisation only gives shadowing values at the $N$ specified locations, shadowing fields give the value of the (potential) shadowing everywhere. This is also useful when $N$ is uncertain, random, or variable.

\section{ACKNOWLEDGMENT}

This work was supported in part by a $P G S D$ award from the National Sciences and Engineering Research Council (NSERC) of Canada.

\section{REFERENCES}

[1] S. R. Hall, A. W. Jeffries, S. E. Avis, and D. D. N. Bevan, "Performance of open access femtocells in 4G macrocellular networks," in WWRF Meeting 20, Apr. 2008.

[2] R. Hekmat and P. V. Mieghem, "Interference power sum with $\log -$ normal components in ad-hoc and sensor networks," in WiOpt, Apr. 2005.

[3] E. Sousa, "Performance of a spread spectrum packet radio network link in a Poisson field of interferers," IEEE Trans. Inf. Theory, vol. 38, no. 6 , pp. 1743-1754, Nov. 1992.

[4] M. Win, P. Pinto, and L. Shepp, "A mathematical theory of network interference and its applications," Proc. IEEE, vol. 97, pp. 205-230, Feb. 2009.

[5] A. Safak and R. Prasad, "Effects of correlated shadowing signals on channel reuse in mobile radio systems," IEEE Trans. Veh. Technol., vol. 40, no. 4, pp. 708-713, Nov. 1991.

[6] T. Klingenbrunn and P. Mogensen, "Modelling cross-correlated shadowing in network simulations," in IEEE VTC, vol. 3, Sept. 1999, pp. $1407-1411$

[7] S. S. Szyszkowicz and H. Yanikomeroglu, "Analysis of interference from large clusters as modeled by the sum of many correlated lognormals," in IEEE WCNC, Mar.-Apr. 2008, pp. 741-745.

[8] B. Alkire, "Cholesky factorization of augmented positive definite matrices," Electrical Engineering Department, UCLA, Tech. Rep., Dec. 2002.

[9] D. Catrein and R. Mathar, "Gaussian random fields as a model for spatially correlated log-normal fading," in IEEE ATNAC, Dec. 2008, pp. $153-157$.

[10] S. S. Szyszkowicz, H. Yanikomeroglu, and J. S. Thompson, "On the feasibility of wireless shadowing correlation models," submitted to IEEE Trans. Veh. Technol., 2010.

[11] N. Beaulieu and Q. Xie, "An optimal lognormal approximation to lognormal sum distributions," IEEE Trans. Veh. Technol., vol. 53, pp. 479-489, Mar. 2004.

[12] K. Kumaran, S. E. Golowich, and S. Borst, "Correlated shadow-fading in wireless networks and its effect on call dropping," Wirel. Netw., vol. 8 , pp. 61-71, Jan. 2002.

[13] I. Forkel, M. Schinnenburg, and M. Ang, "Generation of twodimensional correlated shadowing for mobile radio network simulation," in $W P M C$, vol. 2, Sept. 2004, pp. 314-319.

[14] Z. Wang, E. Tameh, and A. Nix, "Joint shadowing process in urban peer-to-peer radio channels," IEEE Trans. Veh. Technol., vol. 57, pp. 52-64, Jan. 2008.

[15] G. Storvik, A. Frigessi, and D. Hirst, "Stationary space-time Gaussian fields and their time autoregressive representation," Statistical Modelling, vol. 2, pp. 139-161, 2002.

[16] A. Lukin, "Tips \& tricks: Fast image filtering algorithms," in GraphiCon, June 2007, pp. 186-189. 